\title{
EDITORIAL
}

\section{Prudent use of antibiotics: acute exacerbation of COPD as an example}

\author{
G.G.U. Rohde
}

$\mathbf{T}$ his is an important year for respiratory medicine, as 2010 has been declared "Year of the Lung". A multitude of activities have been prepared long in advance and many important initiatives are on their way or yet to come. The European Centre for Disease Prevention and Control (ECDC) has, in parallel, been campaigning for several years for a more prudent use of antibiotics [1]. Last year was devoted to promoting prudent use of antibiotics to primary care prescribers and this year, a campaign promoting prudent antibiotic use to hospital prescribers is well under way [2]. The majority of antibiotics are prescribed for respiratory infections and tuberculosis. The secretary of European Respiratory Society (ERS) Assembly 10 (Respiratory Infections and Tuberculosis) and members of ECDC met recently in order to organise support for European Antibiotic Awareness Day, planned for November 18, 2010. On this date, international respiratory societies also celebrate World COPD Day. This coincidence may be regarded as a signal to illustrate important common aspects of chronic obstructive pulmonary disease (COPD) management and antibiotic awareness.

Antibiotic-resistant strains of pathogenic bacteria are increasingly prevalent in hospitals and the community. Antibiotic prescriptions without clear indication contribute significantly to the increase in antibiotic resistance [3]. Acute exacerbation of COPD (AE-COPD), a disease which has become a leading cause of morbidity and mortality worldwide, often results in administration of antibiotics. For example, in one study, antibiotics were found to have been prescribed in $85 \%$ of 69,820 patients admitted for AE-COPD to 360 hospitals throughout the USA [4].

The evidence for antibiotic prescription for AE-COPD is weak. Recent data suggest that $\sim 70 \%$ of exacerbations are infectious in origin [5]. Of these, only $20-30 \%$ can be related to bacteria detected by sputum culture [5]. It is difficult to prove that pathogens detected in sputum are the relevant triggers of exacerbation, as detection is not proof of infection. Recent findings have shown that growth of bacteria in sputum cultures of COPD patients can also result from colonisation of the airways [6], which is not a clear indication for antibiotic treatment. Moreover, respiratory viruses are detected in $>50 \%$ of exacerbations and also have to be considered as major infectious triggers [7]. In these cases, antibiotic therapy is not effective. Coinfection with bacteria and viruses is prevalent in

CORRESPONDENCE: G.G.U. Rohde, Dept of Respiratory Medicine, Maastricht University Medical Centre (MUMC+), Postbus 5800, 6202AZ Maastricht, The Netherlands. E-mail: g.rohde@mumc.nl half of virus-positive AE-COPD patients and it has not been shown that antibiotic therapy is beneficial in these cases.

In spite of the high prevalence of COPD and the resulting high number of exacerbations worldwide, there is only a very limited number of studies on the efficacy of antibiotic treatment in AE-COPD. A recent Cochrane Review on antibiotics for exacerbations of COPD identified only 11 trials investigating a total of 917 patients [8]. This is in striking contrast to the enormous number of patients suffering from AE-COPD each year worldwide. This recent analysis showed a reduction of the risk of short-term mortality by $77 \%$, a decrease in the risk of treatment failure by $53 \%$ and the risk of sputum purulence by $44 \%$. However, there is a warning that the results have to be interpreted with caution due to the differences in patient selection, antibiotic choice, the small number of trials included and the lack of control for interventions that influence outcome, such as use of systemic corticosteroids and ventilatory support. Most importantly, only three studies (from 1968, 1972 and 2001) with a total of 298 patients were eligible for assessment of mortality. It should be mentioned that, although the combined analysis showed a survival benefit, each individual study was relatively small in size and was underpowered to answer this critical question [9]. The same was true for studies assessing treatment failure (1968, 1972 and 1992; 263 patients) and sputum purulence (1968 and 1972; 205 patients).

There is significant heterogeneity in findings across studies, indicating a lack of consistency and robustness in the results. Recently, a placebo-controlled trial investigated the effects of antibiotic treatment with doxycycline in addition to corticosteroids in AE-COPD [10]. The primary end-point was clinical success on day 30: this was not significantly different between groups. However, secondary end-points, such as clinical success and clinical cure on day 10, were in favour of antibiotic treatment. This study suggests that it is safe to omit antibiotic treatment in a clinical trial of AE-COPD but it cannot answer the crucial question of whether antibiotics are efficacious, as the results are, again, heterogeneous and the study was underpowered. In addition, the antibiotic used, doxycycline, may not be first choice in most European countries. The latest publication in the field is a retrospective cohort study of hospitalised AE-COPD patients performed at 413 acute care facilities throughout the USA [11]. The analysis was based on a database developed for measuring healthcare quality and utilisation. Antibiotic treatment was defined as a minimum of two consecutive days of an antibiotic for AE-COPD. The primary end-point was a composite measure of treatment 
failure, defined as the initiation of mechanical ventilation after hospital day 2, in-hospital mortality and readmission for COPD within 30 days of discharge. It was found that the risk for treatment failure was lower in the antibiotic-treated patients (OR $0.87,95 \%$ CI $0.82-0.92$ ). However, this analysis has many problems. The groups investigated were hardly comparable, as treated patients received more short- and longacting bronchodilators, steroids, morphine and loop diuretics than those not receiving antibiotics. Only administrative data could be used for the analysis, such that physiological differences between groups could not be directly adjusted for. Identification of patients was performed by administrative data review, which implies that classification of patients might have been severely biased. To date, there is no single study sufficiently powered to demonstrate the efficacy of antibiotic treatment in AE-COPD.

It is obvious that there is a paucity of reliable data on the use of antibiotics for AE-COPD. Most of the studies lack an objective definition of COPD and cannot be compared to the standards of current guidelines (such as those of the Global Initiative for Obstructive Lung Disease (GOLD)). Differences in disease definition may have severely biased the results obtained. The different study populations showed clinically important differences in severity of COPD exacerbations. The small number of studies so far does not allow for stratified analysis according to severity of COPD exacerbations. There may also be substantial publication bias, as during the time most of the studies were performed (mainly the 1970s), negative results may not have been published, and such studies were not centrally captured and published. A very important limitation of nearly all studies on the topic is the lack of a standardised treatment for exacerbation. So far, only one clinical trial [10] included standardised treatment, but the results were not unequivocal. However, on this weak basis, antibiotic treatment for AE-COPD is recommended for patients with severe exacerbations or severe underlying COPD. Most episodes of AE-COPD are mild-to-moderate and not severe. At the moment, there is no clear recommendation for antibiotic treatment for these patients. These facts underline the need for a sufficiently powered, randomised, placebo-controlled trial of antibiotics for mild-to-moderate AE-COPD. In the face of the fact that respiratory viruses are detected in $50 \%$ of exacerbations, a major task is the reduction of unnecessary antibiotic treatment in patients with mild-to-moderate AECOPD to stop the threatening increase in antibiotic resistance. It has been convincingly shown that prudent reduction of antibiotic use confers low bacterial resistance without increase in complications [12]. Taking this important aspect into account, it seems that the risk-benefit ratio of antibiotic treatment for mild-to-moderate AE-COPD is negative and such treatment should, therefore, be discouraged. The most recent study [10] showed that it is safe to treat AE-COPD patients of all grades of severity (GOLD I-IV) with placebo if standardised treatment is provided.

Adverse events are an important issue. It has been shown in several trials that adverse events are higher in patients treated with antibiotics than in patients administered a placebo [8]. Hence, the individual patient might benefit from reduced adverse events associated with antibiotic treatment if antibiotics are no longer prescribed for this indication. In addition, the individual patient might also benefit from decreased selection of antibiotic-resistant strains by reduction of antibiotic consumption. Society will clearly benefit if unnecessary antibiotic prescriptions are reduced. This has been convincingly shown in other settings [12].

Finally, there is the potential economic impact. Antibiotic therapy can be costly and it has been shown that reduction in antibiotic use significantly reduces costs [13].

In conclusion, we have to be aware of the fact that, to date, there is no convincing evidence for the use of antibiotics in AE-COPD and clearly, new studies powered enough to definitively show a benefit of these drugs are needed. These studies have to take the heterogeneity of COPD into account, particularly differences in COPD severity, exacerbation frequency and bacterial colonisation. On the current scientific basis, we have to assume that antibiotic treatment of AECOPD contributes significantly to the misuse of antibiotics worldwide. Misuse of antibiotics has been shown to be a major cause of the development of antibiotic-resistant bacteria, a fact that has not been addressed at all in antibiotic studies in AE-COPD so far. Antibiotic resistance itself is a major threat to our patients, as it results in increased morbidity and mortality.

Hence, it is a very positive coincidence that the World COPD Day and the European Antibiotic Awareness Day both take place on November 18, 2010. Let us use this day as a symbol for the urgently needed improvement in COPD care.

\section{STATEMENT OF INTEREST}

None declared.

\section{REFERENCES}

1 Earnshaw S, Monnet DL, Duncan B, et al. European Antibiotic Awareness Day, 2008 - the first Europe-wide public information campaign on prudent antibiotic use: methods and survey of activities in participating countries. Euro Surveill 2009; 14: 19280.

2 European Centre for Disease Prevention and Control. European Antibiotic Awareness Day. http://antibiotic.ecdc.europa.eu Date last accessed: June 30, 2010. Date last updated: 2010.

3 Chen DK, McGeer A, de Azavedo JC, et al. Decreased susceptibility of Streptococcus pneumoniae to fluoroquinolones in Canada. Canadian Bacterial Surveillance Network. N Engl J Med 1999; 341: 233-239.

4 Lindenauer PK, Pekow P, Gao S, et al. Quality of care for patients hospitalized for acute exacerbations of chronic obstructive pulmonary disease. Ann Intern Med 2006; 144: 894-903.

5 Celli BR, Barnes PJ. Exacerbations of chronic obstructive pulmonary disease. Eur Respir J 2007; 29: 1224-1238.

6 Sethi S, Evans N, Grant BJB, et al. New strains of bacteria and exacerbations of chronic obstructive pulmonary disease. $N$ Engl J Med 2002; 347: 465-471.

7 Rohde G, Wiethege A, Borg I, et al. Respiratory viruses in exacerbations of chronic obstructive pulmonary disease requiring hospitalization - a case-control study. Thorax 2003; 58: 37-42.

8 Ram FS, Rodriguez-Roisin R, Granados-Navarrete A, et al. Antibiotics for exacerbations of chronic obstructive pulmonary disease. Cochrane Database Syst Rev 2006; 2: CD004403.

9 Quon BS, Gan WQ, Sin DD. Contemporary management of acute exacerbations of COPD: a systematic review and metaanalysis. Chest 2008; 133: 756-766. 
10 Daniels JM, Snijders D, de Graaff CS, et al. Antibiotics in addition to systemic corticosteroids for acute exacerbations of COPD. Am J Respir Crit Care Med 2010; 181: 150-157.

11 Rothberg MB, Pekow PS, Lahti M, et al. Antibiotic therapy and treatment failure in patients hospitalized for acute exacerbations of chronic obstructive pulmonary disease. JAMA 2010; 303: 2035-2042.
12 Molstad S, Erntell M, Hanberger H, et al. Sustained reduction of antibiotic use and low bacterial resistance: 10-year follow-up of the Swedish Strama programme. Lancet Infect Dis 2008; 8: 125-132.

13 Bantar C, Sartori B, Vesco E, et al. A hospitalwide intervention program to optimize the quality of antibiotic use: impact on prescribing practice, antibiotic consumption, cost savings, and bacterial resistance. Clin Infect Dis 2003; 37: 180-186. 